\title{
Aplicación Web para Niños con Problemas de Dicción
}

\section{Web Application for Children with Diction Problems}

RODRÍGUEZ-VARGAS, María de Jesús广๋*, VILLANUEVA-GARCÍA, Guadalupe, GÓMEZGARCÍA, Arnulfo y ARROYO-ALMAGUER, Marisol

Universidad Tecnológica del Suroeste de Guanajuato, Tecnologías de la Información y Comunicación

ID $1^{\mathrm{er}}$ Autor: María De Jesús, Rodríguez-Vargas

ID $1^{\text {er }}$ Coautor: Guadalupe, Villanueva-García

ID $2^{\text {do }}$ Coautor: Arnulfo, Gómez-García

ID $3^{\text {er }}$ Coautor: Marisol, Arroyo-Almaguer

DOI: $10.35429 /$ JCS.2019.10.3.1.5

Recibido: 12 de Agosto, 2019; Aceptado 30 de Diciembre, 2019

\section{Resumen}

La aplicación web para niños con problemas de dicción, busca contribuir a la disminución de un problema de pronunciación conocido como la dislalia. La aplicación puede emplearse en cualquier dispositivo conectado a internet, su principal propósito es a través de ejercicios y dinámicas integrados en la misma, mejorar la dicción que presentan los niños en el rango de 4 a 6 años de edad. La metodología empleada es la de desarrollo de iteraciones que permite reducir el riesgo que surge entre las necesidades del usuario y el producto final por malos entendidos durante la etapa de recolección de requisitos; al tener la ventaja de realizar el desarrollo en pequeños ciclos, permitiendo gestionar mejor los riesgos y las entregas. La aplicación web para niños con problemas de dicción, en su primera versión, ha tenido buenos resultados, destacando su funcionalidad y aplicación, que ha mostrado gran aceptación por parte de los niños y niñas, educadores, expertos e incluso padres de familia, pero sobre todo porque en el lapso de uso de un mes se han comenzado a tener mejoras en la pronunciación de los usuarios (niños y niñas).

Aplicación web, Dislalia, Problemas de dicción

\begin{abstract}
The web application for children with diction problems, seeks to contribute to the reduction of a pronunciation problem known as dyslalia. The application can be used on any device connected to the internet, its main purpose is through exercises and dynamics integrated in it, improve the diction that children present in the range of 4 to 6 years of age. The methodology used is the development of iterations that allows reducing the risk that arises between the needs of the user and the final product due to misunderstandings during the requirements collection stage; having the advantage of carrying out the development in small cycles, allowing to better manage risks and deliveries. The web application for children with diction problems, in its first version, has had good results, highlighting its functionality and application, which has shown great acceptance by children, educators, experts and even parents, but about all because in the period of use of a month have begun to have improvements in the pronunciation of users (boys and girls).
\end{abstract}

Web application, Dyslalia, Diction problems

Citación: RODRÍGUEZ-VARGAS, María de Jesús, VILLANUEVA-GARCÍA, Guadalupe, GÓMEZ-GARCÍA, Arnulfo y ARROYO-ALMAGUER, Marisol. Aplicación Web para Niños con Problemas de Dicción. Revista de Simulación Computacional. 2019. 3-10: 1-5

$\dagger$ Investigador contribuyendo como primer autor. 


\section{Introducción}

En el bullying suelen ser más propensos aquellos niños que poseen diversidad funcional; los niños que tienen problemas de dicción muestran como consecuencia del acoso, retraimiento al hablar, por la pronunciación que tienen. Por ello, tratan de evitar las palabras que se les dificultan, hablar en público, etc. volviéndose tímidos e inseguros de sí mismos.

La dislalia es una discapacidad para pronunciar correctamente ciertos fonemas o grupos de fonemas debido al establecimiento de puntos de articulación erróneos, los cuales deben ser considerados anormales después de los 5 años. Existen diversas aplicaciones dirigidas a atender esta problemática, entre ellas: "Maletín de la R", "AppLalia" y "Vocalización y dicción", sin embargo, no cuentan con las herramientas necesarias para disminuir significativamente el problema, ya que su material es poco atractivo, escaso y repetitivo.

La propuesta que se presenta es una aplicación web desarrollada a la cual se podrá acceder desde cualquier dispositivo que tenga conexión a internet, la página web es responsiva (ajustable a cualquier tamaño de pantalla), está integrada por actividades como juegos y videos que pretenden hacer el aprendizaje dinámico y divertido, los ejercicios que se utilizan dentro de la aplicación están orientados para ayudar a mejorar la dicción en los niños.

Como se menciona previamente, se busca mejorar la pronunciación (en esta primera versión) de la $r$ a través del juego volviendo el aprendizaje divertido; evitando que los padres de familia o profesores empleen diferentes sitios o herramientas, cuando es posible tenerlos en una sola aplicación, con un diseño atractivo e intuitivo. Sus diferentes interfaces permiten luego del acceso, la realización de actividades como test, visualización de videos, etc., es decir diferentes técnicas y ejercicios.

\section{Hipótesis}

Utilizar la aplicación como parte del proceso de recuperación de puntos de articulación en niños en el rango de 4-6 años de edad, permitirá que aprendan jugando al realizar ejercicios de motricidad-bucofacial y se sientan seguros de comunicarse en cualquier entorno.

\section{Desarrollo}

\section{Marco referencial}

Existen diversas aplicaciones dirigidas a atender esta problemática, por lo que, para el desarrollo del presente proyecto, se revisaron aplicaciones, plataformas, proyectos de investigación, etc., destacando:

- El maletín de la $\mathrm{R}$ es un material destinado a la corrección de los errores cometidos en la pronunciación del fonema $/ \mathrm{r} /$, (rotacismo), en su forma simple o múltiple. El método se desarrolla a través de un tratamiento directo, uno indirecto, actividades lúdicas y actividades de adquisición del fonema haciendo uso de otros ya adquiridos. El material dispone de una App para dispositivos móviles y tabletas que complementa y refuerza aspectos como: la discriminación auditiva; la repetición o emisión ecoica de fonemas, palabra y/o frases; la audición de sonidos y discriminación de los mismos; la reproducción fonológica por medio de sílabas o imágenes; la mejora del léxico específico; completado de oraciones con ayuda de tiras pictográficas; afianzamiento de la lectura (Gaspar González, 2016).

AppLalia es un juego desarrollado para mejorar el trastorno de la articulación de los fonemas, en la que el niño o niña no es capaz de repetir por imitación las palabras que escucha y lo hace de forma incorrecta desde el punto de vista fonético. ejemplo: "Perro - Pelo" la aplicación realiza una seria de ejercicios interactivos para para corregir este trastorno (Blandon, 2017).

Vocalización y dicción, busca que se aprenda a vocalizar y a utilizar las palabras con ejercicios de dicción. En esta aplicación de Vocalización y Dicción para dispositivos móviles y tabletas, se integra un conjunto de los mejores vídeos con las técnicas y consejos seleccionados por especialistas logopedas para superar las dificultades de comunicación de niños y adultos (Entertainment, 2015). 


\section{Metodología a desarrollar}

La metodología de iteraciones permite analizar los avances de cada fase, reducir el riesgo que surge entre las necesidades del usuario y el producto final por malos entendidos durante la etapa de recolección de requisitos; ya que al realizar el desarrollo en pequeños ciclos, posibilita gestionar mejor los riesgos y las entregas; mantener una retroalimentación constante y manejo de cambios (flexibilidad).

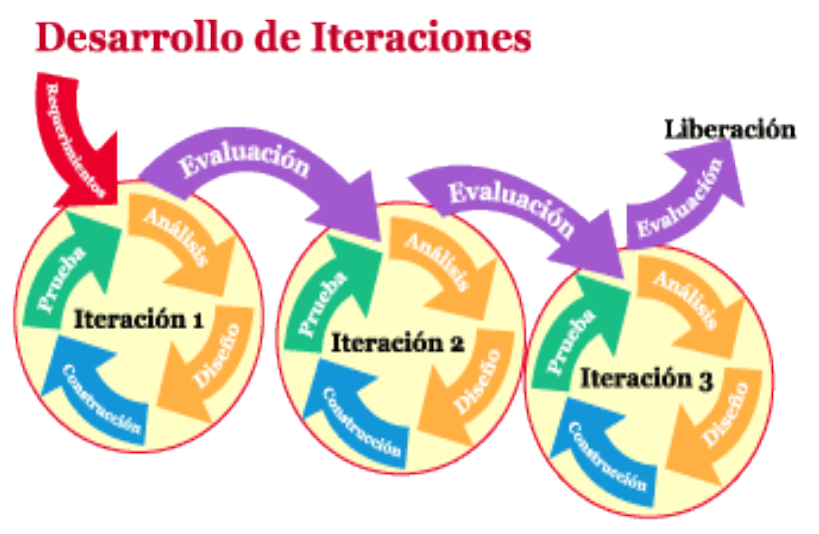

Figura 1 Modelo de desarrollo

Fuente: Imagimática - Consultores en sistemas y nuevos medios.

http://www.imagimatica.com.mx/desarrollo_software.ht $m l$

Requerimientos: Para la fase de requerimientos se emplea junto con la investigación documental, la entrevista con personas que enfrentan este problema, así como con profesores de educación básica.

Análisis: Una vez obtenidos los requerimientos se realizó el análisis de los mismos, determinando las funciones que debe abordar la aplicación, siendo las siguientes: dinámicas, videos, juegos y niveles que vayan motivando el aprendizaje.

Diseño: Se identificaron 3 tipos de usuario, como se puede apreciar en el siguiente diagrama de caso de uso

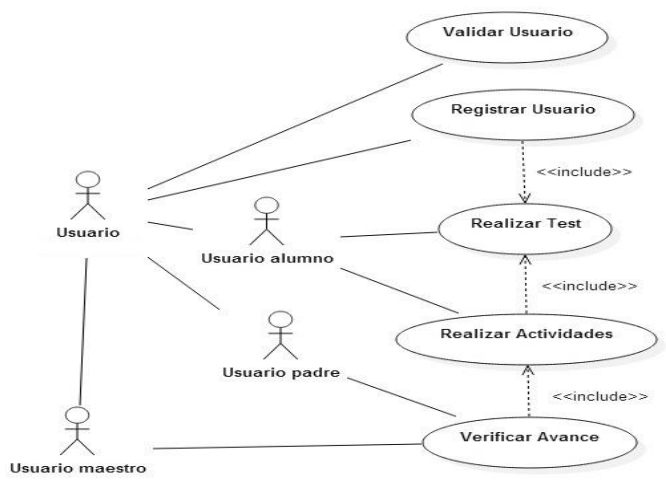

Figura 2 Caso de uso

Fuente: Elaboración Propia

Construcción: DICAP implementa el uso de PHP, CSS, HTML en su construcción.

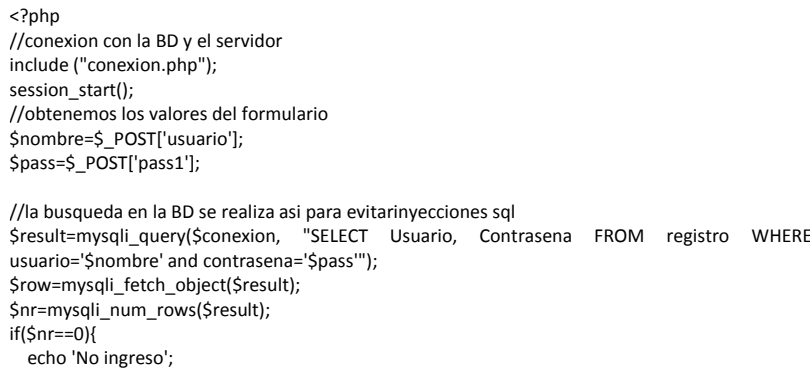

Figura 3 Fragmento de código

Fuente: Elaboración Propia

Pruebas: la aplicación se ha probado en diferentes escenarios, tales como exposiciones de proyectos al interior de la Universidad, en foros y en dos instituciones de nivel preescolar; donde se ha tenido presencia de educadores, niños y padres de familia, obteniendo importantes oportunidades de mejora y gran aceptación por su fácil manejo.

\section{Resultados}

Actualmente la aplicación se encuentra en su versión 1.0 la cual se integra por:

1. Pantalla de inicio. El usuario debe registrarse para tener acceso a la plataforma. 


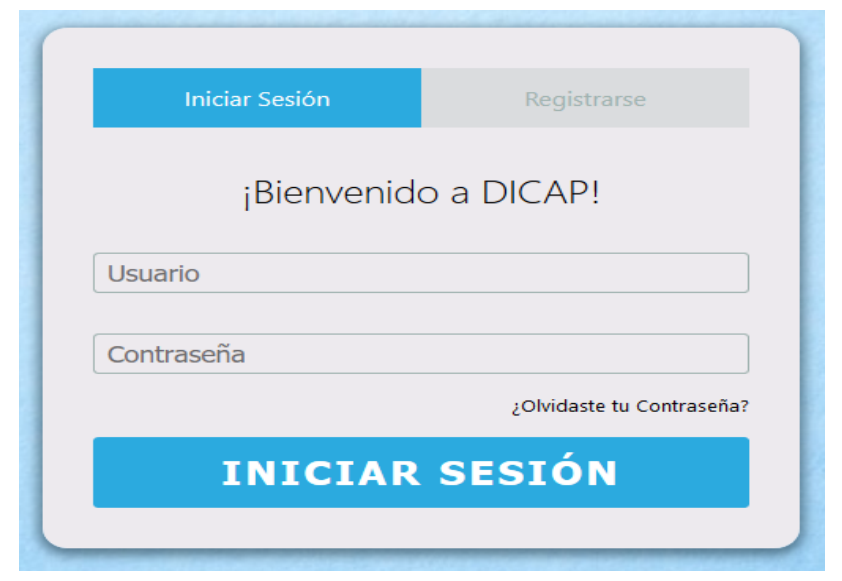

Figura 4 Pantalla de inicio

Fuente: Elaboración Propia

2. Pantalla de elección de roles para el registro. Muestra los distintos roles con los que cuenta el sistema.

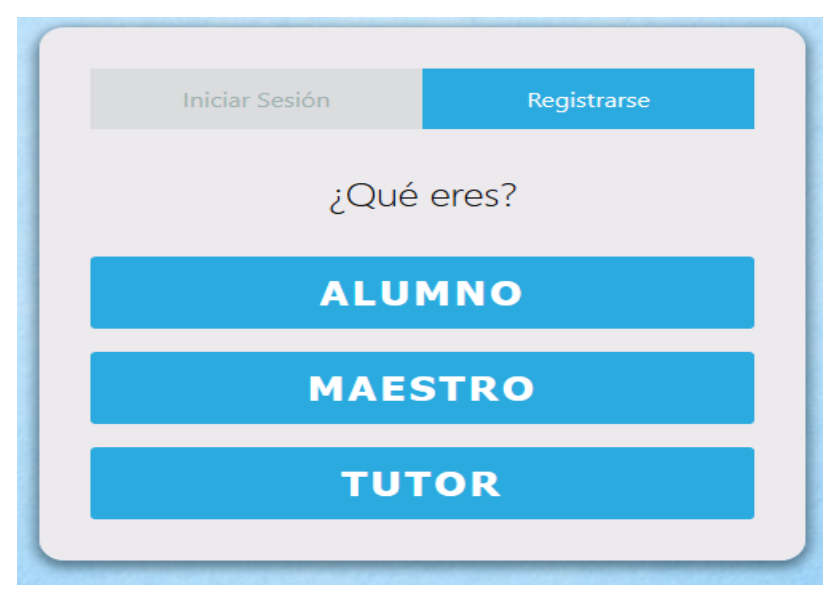

Figura 5 Pantalla de elección de roles Fuente: Elaboración Propia

3. Pantalla con la solicitud de datos para darse de alta en la plataforma. Muestra algunas preguntas para darse de alta en el sistema.

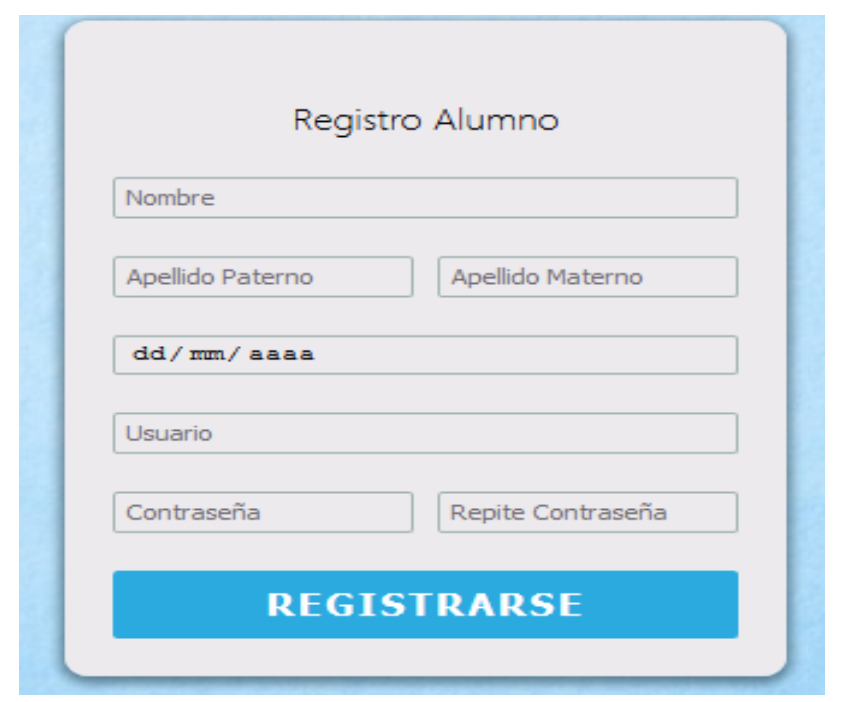

Figura 6 Pantalla de registro

Fuente: Elaboración Propia
4. Pantalla principal de la aplicación con el rol de alumno. Muestra brevemente información de la aplicación para comenzar a utilizarla y conocerla.

5. Pantalla de videos. Muestra videos de ejercicios bucofaciales.

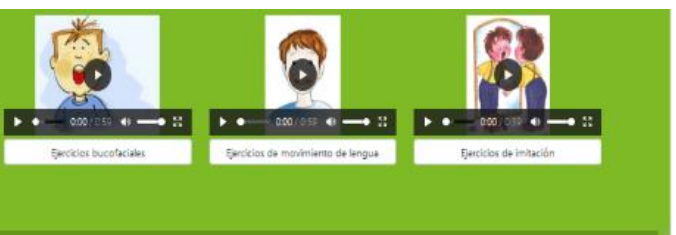

Figura 7 Pantalla de videos

Fuente: Elaboración Propia

6. Pantalla de juegos. Muestra una serie de juegos que ayuden al alumno.

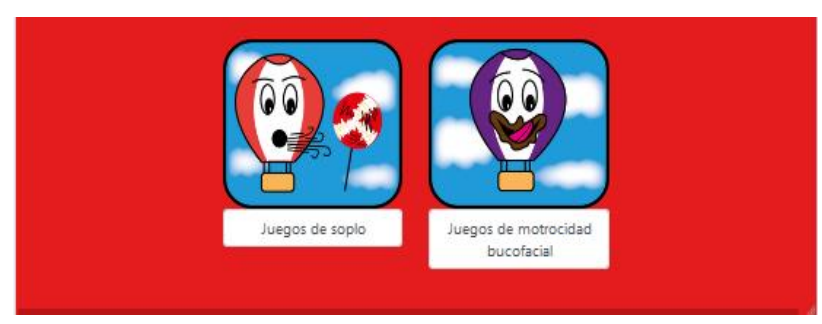

Figura 8 Pantalla de juegos

Fuente: Elaboración Propia

7. Pantalla Nosotros. Muestra una pequeña descripción acerca de la aplicación web y los autores.

8. Pantalla del Maestro. Muestra los alumnos que tiene registrados.

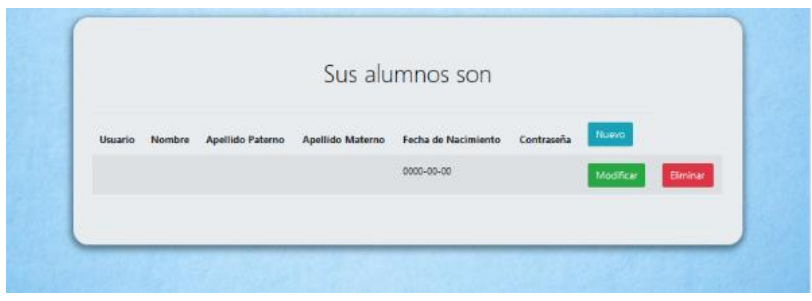

Figura 9 Pantalla del maestro

Fuente: Elaboración Propia

9. Pantalla del tutor. Muestra los tutorados que tiene a su cargo.

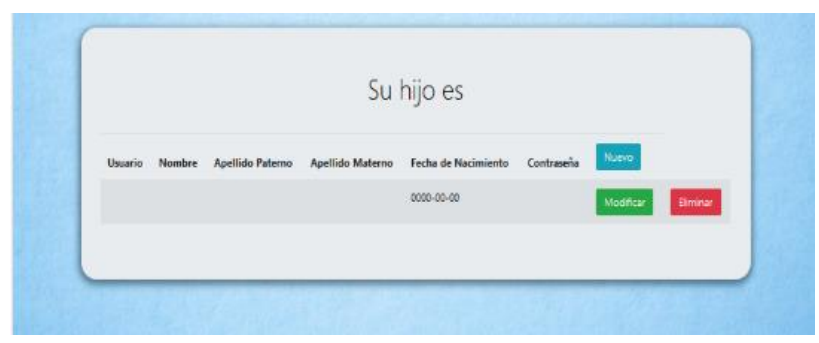

Figura 10 Pantalla del tutor Fuente: Elaboración Propia

RODRÍGUEZ-VARGAS, María de Jesús, VILLANUEVA-GARCÍA, Guadalupe, GÓMEZ-GARCÍA, Arnulfo y ARROYO-ALMAGUER, Marisol. Aplicación Web para Niños con Problemas de Dicción. Revista de Simulación Computacional. 2019 


\section{Agradecimiento}

Por las facilidades y apoyo brindados:

A la Universidad Tecnológica del Suroeste de Guanajuato a través de la carrera de Tecnologías de la Información y Comunicación.

Al Jardín de Niños María Montessori de Valle de Santiago, Gto.

A la Escuela Primaria Justo Sierra de la Comunidad de Mogotes, Municipio de Valle de Santiago, Gto.

Padres de familia de la Comunidad de Mogotes de San José Parangueo, Municipio de Valle de Santiago, Gto.

\section{Conclusiones}

La aplicación web para niños con problemas de dicción, es un proyecto ambicioso que busca convertirse en una herramienta tecnológica innovadora en el área de la educación básica, específicamente para niños con problemas de pronunciación. Los resultados hasta ahora obtenidos muestran una gran aceptación, versatilidad y aplicabilidad en los diferentes contextos, considerando que las generaciones actuales están estrechamente ligadas al uso de dispositivos tecnológicos de vanguardia; lo que seguramente se traduce en una nueva forma de adquirir habilidades de motricidad bucofacial.

El empleo de este tipo de herramientas por parte de los formadores, resulta ser un instrumento valioso en su labor de enseñanza y de terapia, al utilizar tecnología que les permita interactuar con sus grupos y mejorar el proceso del rendimiento.

\section{Referencias}

A. Nevárez-Racon, JA Medina-Lopéz, MM Nevárez-Rascón, J. Vargaz-Esquivel,G. De la Torre-Morán y A. Donohué-Cornejo. (2013). Atención al rhotacismo.GEU, E. (23 de Febrero de 2016). Google Play. Obtenido de Google Play:

https://play.google.com/store/apps/details?id=c om.editorialgeu.praxiasr\&hl=es_419.

Blandon, A. K. (2017 de agosto de 18). APPLALIA. Obtenido de https://play.google.com/store/apps/details?id=c om.andros.kent.applalia
ELENA, U. E. (2013). Obtenido de http://repositorio.upse.edu.ec/bitstream/46000/1 028/1/proyecto\%20HILDA2.pdf

Entertainment, A. \&. (2015 de julio de 19). Vocalización y dicción. Obtenido de https://play.google.com/store/apps/details?id=c om.frenchie.vocalizacion.diccion

Gaspar González Rus, I. M. (10 de marzo de 2016). El maletín de la R. Obtenido de https://play.google.com/store/apps/details ?id=c om.editorialgeu.maletinR\&hl=es_MX

H. Andrade, L. P. (2014). Programa psicopedagógico de detección, asistencia y estimulación lingüística de problemas de dislalia en niños de jardines infantiles de la ciudad de Sucre. (M.Ramos, Ed.) Ciencias Sociales Handbooks.

http://www.consumer.es/web/es/educacion/extr aescolar/2006/04/13/150965.php?page $=2$

López, D. N. (2002;). Obtenido de http://www.sld.cu/galerias/pdf/sitios/rehabilitac ion-logo/anquiloglosia_.pdf.

Odontostomat, J. (2013). scielo. Obtenido de http://www.scielo.cl/scielo.php?pid=S0718381X2013000100004\&script=sci_arttext

Rey Millan, M. (2015). rodin. Obtenido de http://rodin.uca.es/xmlui/handle/10498/18375 